\title{
Optimization of Ion Implantation Condition for Depletion-type Silicon optical modulators
}

\author{
Hui Yu, Wim Bogaerts, Member, IEEE, and An De Keersgieter
}

\begin{abstract}
We study the influence of the ion implantation conditions on the performance of depletion-type silicon optical modulators by a combined simulation of the process flow, the electrical characteristic and the beam propagation. Through calculations about different implantation positions, energies and tilt angles, our work reveals that a gap between specific implantation windows is able to alleviate the modulation efficiency degradation due to the lateral straggling of implanted ions, while a tilt angle reduces the optical loss without harming the modulation efficiency. After an optimization about the implantation condition, the extinction ratio of the Mach-Zehnder modulator can be improved by $4.6 \mathrm{~dB}$, while its optical loss falls from $3 \mathrm{~dB}$ to $2.47 \mathrm{~dB}$. Finally, a simplified doping pattern which eliminates 2 implantation steps is discussed.
\end{abstract}

Index Terms-Device modeling, ion implantation, optical modulation, plasma-dispersion effect, silicon photonics.

\section{INTRODUCTION}

$\mathrm{O}$ ptical modulators which encode a bit stream onto the optical carrier are an essential functional component for any optical communication links ranging from telecommunications to optical interconnects. Among different modulation techniques, all-silicon modulators are promising due to their compatibility with mature CMOS technology. Since unstrained silicon exhibits no linear electro-optic effect [1] and only very weak Kerr and Franz-Keldysh effects, most high-speed silicon modulators rely on the carrier plasma dispersion effect [2], where a change in carrier concentration causes a change in absorption, and through the Kramer-Kronig relations, a change in refractive index. A carrier density variation can be reached by carrier injection in a forward biased p-i-n diode [3]-[5], accumulation in a MOS capacitor [6], [7] or depletion in a reverse biased p-n diode [8]-[13]. Among the three mechanisms, carrier depletion has an advantage on operation speed since it relies on the electric-field induced majority carrier dynamics. However, it suffers from low modulation efficiency due to a relatively small overlap between the carrier depletion region and the optical mode. A lot of efforts are directed to improve its modulation efficiency, such

Manuscript received February 3, 2010.

H. Yu and W. Bogaerts are with the Photonics Research Group, Ghent University - imec, Department of Information Technology (INTEC), Sint-Pietersnieuwstraat 41, 9000 Gent, Belgium (e-mail: hyu@intec.ugent.be). W. Bogaerts acknowledges the Flemish Research Foundation for a postdoctoral fellowship.

A. D. Keersgieter is with imec, 3001, Leuven, Belgium. as increasing the doping concentration [9], utilizations of ring resonators [10], [11], or enhancing the overlap between the optical mode and the depletion region by interleaved $p-n$ junctions perpendicular to the light propagation direction [8].

Besides improving the depletion-type modulator by better optical or electrical designs, we should also pay attention to the influence of practical processing conditions. A key step to manufacturing any depletion-type modulators is to implement p-n diodes inside the optical waveguide. In most cases this is achieved by ion implantation, which directly determines the doping profile and the consequent modulation characteristic. On the other hand, a variety of parameters that compose a practical implantation offer us a large space for a process optimization. In this paper, we simulate and optimize the implantation condition for depletion-type optical modulators with the aid of a commercially available process simulator which is able to model whole semiconductor manufacturing processes. This paper is organized as follows: In section II, we present our design of a typical abrupt p-n junction based optical phase shifter, which serves as a target structure of the following process optimization. In section III, we study the influence on the optical modulation of different implantation parameters, such as implantation position, energy and tilt angle. In section IV, we reduce 2 ion implantation steps without affecting the performance of the device. Finally a conclusion is reached in section $\mathrm{V}$.

\section{DeVICE Design}

Fig. 1(a) shows a schematic cross section of an ideal abrupt p-n junction based optical phase shifter. It is a shallow-etched rib waveguide with an embedded lateral $\mathrm{p}$-n diode based on the silicon-on-insulator (SOI) platform. A phase difference would accumulate with respect to a reference waveguide as a beam passes through the phase shifter. A Mach-Zehnder interferometer then converts the phase difference into the final optical intensity modulation. The rib width and height are 500 $\mathrm{nm}$ and $220 \mathrm{~nm}$ respectively, while the etching depth is $70 \mathrm{~nm}$ for the compatibility with grating-based fiber coupling structures [14]. Two moderately doped slits with equal concentration $N$ and opposite polarities form a p-n junction inside the rib waveguide. A driven voltage sweeps out free carriers inside the two slits, which compose an active region of the modulator. Since a density variation of holes induces a larger refractive index change than that of electrons, there is a 


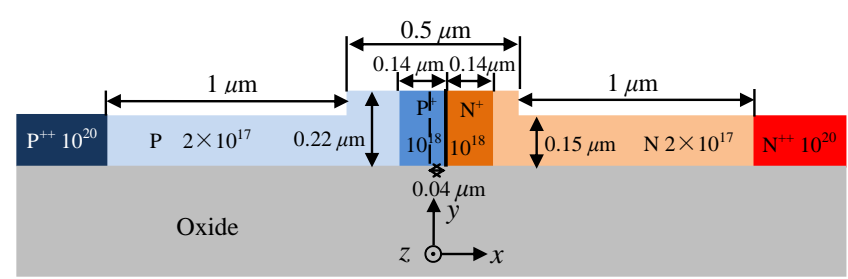

(a)

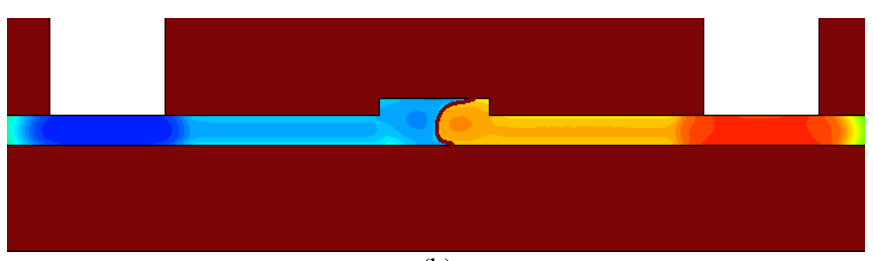

(b)

Fig. 1. (a) Schematic cross section of an ideal abrupt p-n junction based optical phase shifter. (b) Practical dopant distribution achieved by the implantation condition in Table. 1.

small offset between the junction plane and the optical mode center. The optimal value is $0.04 \mu \mathrm{m}$ for our structure in Fig. $1(\mathrm{a})$.

According to the optical waveguide theory, the effective mode index change $\Delta n_{\text {eff }}$ of a rib waveguide is proportional to the overlap integral between the optical mode and the local refractive index change $\Delta n(x, y)$ over the whole carrier depletion region:

$$
\Delta n_{\mathrm{eff}}=\frac{\iint_{s} \Delta n(x, y)|E(x, y)|^{2} d x d y}{\iint_{s}|E(x, y)|^{2} d x d y}
$$

Where $E(x, y)$ is the the electric field distribution of the optical mode. For the lateral p-n junction in Fig. 1(a), we only consider the integral in $x$ direction. Normally the scale of the optical mode is much larger than that of the depletion region (electrical field $1 / \mathrm{e}$ width of $0.85 \mu \mathrm{m}$ for the rib waveguide here), thus the optical intensity can be regarded as a constant throughout the depletion region for a first approximation. Therefore, the effective mode index change $\Delta n_{\text {eff }}$ is approximately proportional to the product of the doping concentration $N$ and the depletion region width $w: \Delta n_{\text {eff }} \propto w N$.

On the other hand, the depletion region width is reverse proportional to the square root of the doping concentration: $w \propto 1 / \sqrt{N}$ [15], so finally we can deduce that under a certain reverse bias the effective mode index change is proportional to the square root of the doping concentration: $\Delta n_{\text {eff }} \propto \sqrt{N}$. This relationship implies that we can enhance the modulation efficiency by increasing the doping concentration in the active region. Ideally, the active region can be made equal to the depletion region. Any excess of the active region over the depletion region does not contribute to the effective mode index change but increases the optical loss. However, the depletion region shrinks with increasing the doping concentration, while the smallest slit width is always limited by the practical lithography technology, so we cannot increasing the doping concentration in the active region without any limit. For our optical lithography system ASML PAS5500/1100 which uses an ArF laser at $193 \mathrm{~nm}$ for imaging, the smallest reliable wire width is about $140 \mathrm{~nm}$ [16]. We choose this value as the slit width and set a doping concentration of $10^{18} / \mathrm{cm}^{3}$. A simple calculation shows that the depletion region inside both $n$ and $p$ type materials is $84.2 \mathrm{~nm}$ at a reverse bias of $-10 \mathrm{~V}$, which is much less than the slit width of $140 \mathrm{~nm}$. We will discuss how to remedy this issue by a proper implantation condition in section III. In order to reduce the optical absorption loss, two heavily doped regions $\left(10^{20} / \mathrm{cm}^{3}\right)$ to form Ohmic contacts are situated 1 $\mu \mathrm{m}$ apart from the rib, and the two regions connecting the contact regions and the slits are lightly doped to $2 \times 10^{17} / \mathrm{cm}^{3}$. We will give an explanation about this value in section III.

\section{IMPLANTATION CONDITION OPTIMIZATION}

In this section we simulate how an implantation condition would impact the modulation characteristic. Before any specific simulation, it is necessary to introduce the whole simulation flow: At first, a two-dimensional process simulator DIOS is employed to model the fabrication sequences of the modulator [17], which contains waveguide etching, ion implantation, annealing and contact metallization. The program employs analytic implantation models and state-of-the art point defect models that are calibrated to a large number of experiments to model ion implantation and diffusion. It generates files which contain exact impurity distribution after ion implantation and annealing. In our simulation, a dual primary distribution function which consists of two Pearson-IV distributions is selected to take the ion channeling effect into account [18]. The lateral straggling of implanted ions is specified by a Gaussian function with a depth-dependent standard deviation. A pair diffusion model is chosen to calculate the dopant diffusion in the rapid thermal annealing process, which is $1050{ }^{\circ} \mathrm{C}$ for $10 \mathrm{~s}$. After creating a virtual modulator by DIOS, we use a two-dimensional device simulation package DESSIS to calculate its electrical characteristic [17]. The program solves the coupled Poisson equation and the charge continuity equation for electrons and holes numerically. Its output results include carrier distributions at different biases, transient responses to given driven signals, and a small-signal equivalent circuit. Following semiconductor physics models are employed in our calculations:

- the Shockley-Read-Hall (SRH) recombination model with doping-dependent lifetime,

- the mobility model which includes the doping dependence effect and the high field saturation effect,

- the silicon band-gap narrowing model.

The carrier distribution is then converted to the distribution of refractive index through the free-carrier dispersion relationship at $1.55 \mu \mathrm{m}$ [2]. Finally an optical model solver is used to calculate effective model index shifts and optical losses of the phase shifter at different bias voltages.

Table 1 presents an un-optimized implantation condition to 
TABLE I

An IMPLANTATION CONDITION Without ANy OPTIMIZATION

\begin{tabular}{ccccc}
\hline \hline & $\begin{array}{c}\text { Implantation } \\
\text { window }\end{array}$ & Species & Dose & Energy \\
\hline $\mathrm{P}++$ & $(-1.8,-1.25)$ & $\mathrm{B}$ & $1.5 \times 10^{15} / \mathrm{cm}^{2}$ & $20 \mathrm{kev}$ \\
$\mathrm{P}$ & $(-1.25,-0.1)$ & $\mathrm{B}$ & $0.3 \times 10^{13} / \mathrm{cm}^{2}$ & $20 \mathrm{kev}$ \\
$\mathrm{P}+$ & $(-0.1,0.04)$ & $\mathrm{B}$ & $2.2 \times 10^{13} / \mathrm{cm}^{2}$ & $30 \mathrm{kev}$ \\
$\mathrm{N}+$ & $(0.04,0.18)$ & $\mathrm{P}$ & $2.2 \times 10^{13} / \mathrm{cm}^{2}$ & $100 \mathrm{kev}$ \\
$\mathrm{N}$ & $(0.18,1.25)$ & $\mathrm{P}$ & $0.3 \times 10^{13} / \mathrm{cm}^{2}$ & $70 \mathrm{kev}$ \\
$\mathrm{N}++$ & $(1.25,1.8)$ & $\mathrm{P}$ & $1.5 \times 10^{15} / \mathrm{cm}^{2}$ & $70 \mathrm{kev}$ \\
\hline \hline
\end{tabular}

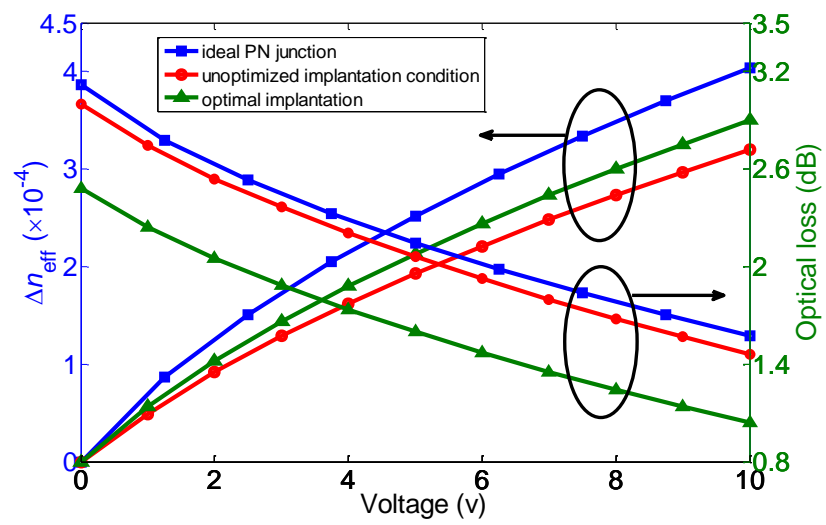

(a)

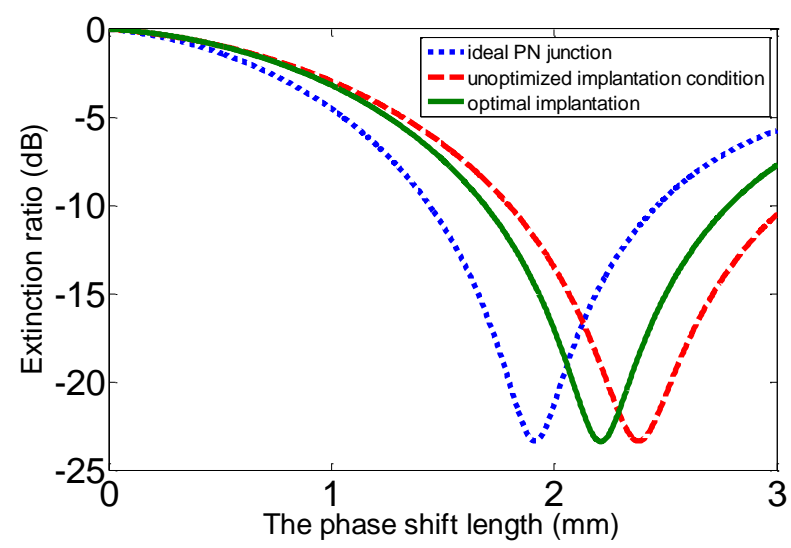

(b)

Fig. 2. Performances of modulators with different doping profiles. (a) The effective mode index change and the optical loss as a function of the reverse bias. The optical loss of the phase shifter at $0 \mathrm{~V}$ is specified for a device length that leads to $\pi$ phase shift at a reverse bias of $10 \mathrm{~V}$. (b) The extinction ratio of the modulator at a reverse bias of $10 \mathrm{~V}$ versus the phase shifter length. Only the free carriers absorption loss is considered in our calculation.

approach the ideal structure presented in Fig. 1. Implantation positions are mapped directly from the ideal impurity distribution of the target structure. The origin of the coordinates in Table. I locates at the waveguide center. Doping doses are determined by the product of the target doping concentration and the thickness of local silicon layer. Different implantation energies make concentration peaks of impurities locate in the center of the local Si layer. The final impurity distribution and the corresponding junction position are shown in Fig. 1(b). Because of the inevitable lateral straggling of implanted ions, we could only get a gradient junction which contains a transition region where two dopants meet and compensate each other. Inside this region the net dopant concentration is much smaller than the design value. As we have mentioned in section II , a low net doping concentration would weaken the modulation efficiency. This point is clear in Fig. 2(a), which presents the effective mode index change and the optical loss as a function of the reverse bias for 3 doping profiles. We can see that the effective mode index change at $10 \mathrm{~V}$ falls from $4.04 \mathrm{e}-4$ to $3.2 \mathrm{e}-4$ with the implantation condition in Table. I, consequently the extinction ratio of the modulator falls from $23.3 \mathrm{~dB}$ to $11.6 \mathrm{~dB}$ provided that the phase shifter length is 1.9 $\mathrm{mm}$ as shown in Fig. 2(b).

Although the lateral straggling of implanted ions is inevitable, the detriment of it can be alleviated in some extent by proper positions of relevant implantation windows. To illustrate this point qualitatively, we make a linearly graded junction assumption here, under which the depletion width $w$ is reverse proportional to the cube root of the doping gradient: $w \propto a^{-1 / 3}$ [15]. Still neglecting the optical intensity variation inside the depletion region, we have $\Delta n_{\text {eff }} \propto \int_{0}^{w} a x d x \propto a^{1 / 3}$, which means a steep junction is preferable. If we approximate the lateral straggling of implanted ions by Gaussian functions, the net dopant concentration in the transition region is the difference between two Gaussian distributions. Theoretically it can be deduced that if the distance between the two Gaussian peaks is equal to the sum of their standard deviation, the net dopant concentration has the largest gradient at its zero point. As a consequence the modulator would have the highest modulation efficiency. This can be confirmed by the simulation result in Fig. 3, which presents the performance of the phase shifter as a function of the implantation position for two doping doses. In this simulation, the $\mathrm{P}+$ and $\mathrm{N}+$ implantation regions are $\left(0.04-x-w_{\mathrm{I}}, 0.04-x\right)$ and $\left(0.04+x, 0.04+x+w_{\mathrm{I}}\right)$ respectively, where $w_{\mathrm{I}}$ is the width of the implantation window. The two implantation windows with a gap of $2 x$ are symmetrical about the plane $x=0.04 \mu \mathrm{m}$ in order to form a p-n junction here. Implantation energies here are the same as those listed in Table. I, the resulting average lateral standard deviations of $\mathrm{B}$ and $\mathrm{P}$ are $\sigma_{\mathrm{B}}=43 \mathrm{~nm}$ and $\sigma_{\mathrm{P}}=36 \mathrm{~nm}$ respectively [18]. In Fig. 3 increasing the implantation dose does enhance the modulation efficiency as expected in section II . Fig. 3 also indicates that the optimal gap between the $\mathrm{N}+$ and $\mathrm{P}+$ windows is $40 \mathrm{~nm}$ for both doping doses, which is smaller than the value of $\left(\sigma_{\mathrm{B}}+\sigma_{\mathrm{P}}\right)$. The reason for this deviation is that the start point of a Gaussian-type lateral straggling always locates several tens of nanometers inside the corresponding implantation window. Since a proper gap between the $\mathrm{N}+$ and $\mathrm{P}+$ implantation windows benefits the modulation efficiency, we should take this point into account when designing the mask. Figure 3 also provides an insight into the impact of lithography alignment errors. Any misalignments between the $\mathrm{P}+$ and $\mathrm{N}+$ implantation windows can weaken the modulation efficiency according to the date in Fig. 3, so high resolution lithography is critical for such devices. 


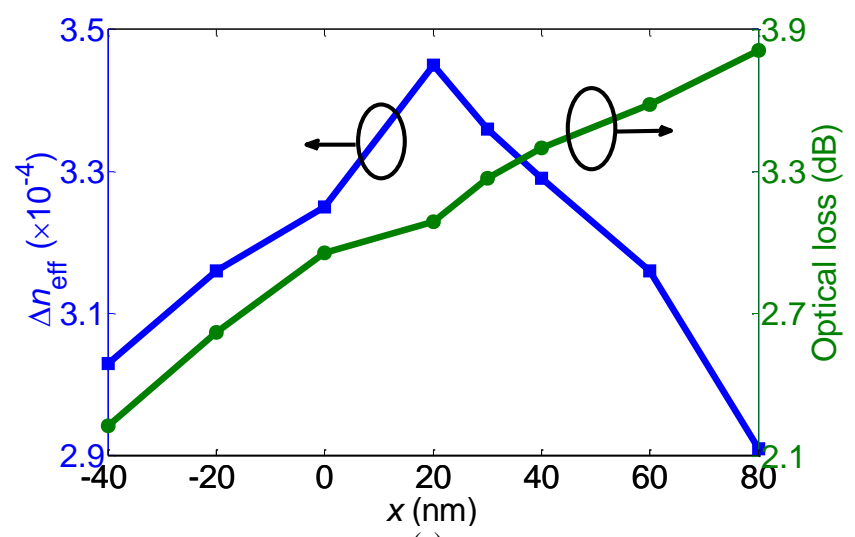

(a)

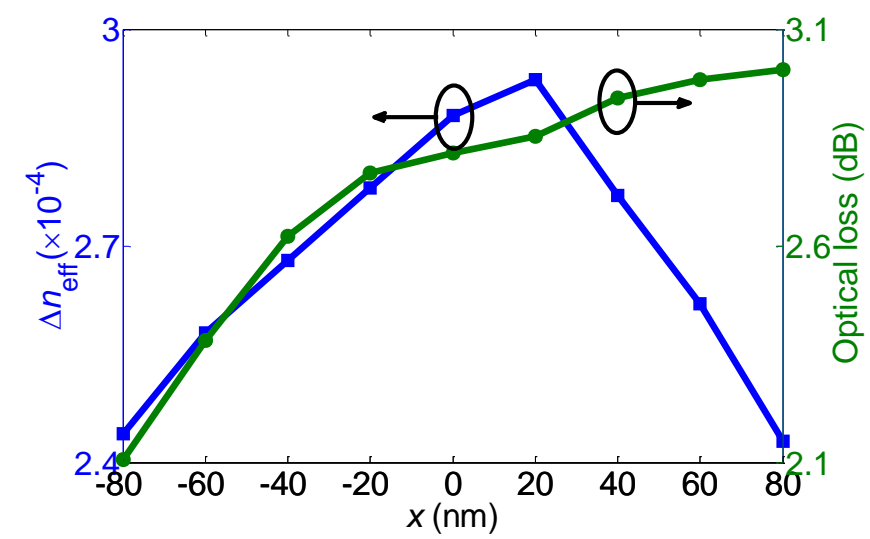

(b)

Fig. 3. Performance of the phase shifter versus the positions of the $\mathrm{N}+$ and $\mathrm{P}+$ implantation windows. (a) Dose $=2.2 \times 10^{13} / \mathrm{cm}^{2}, w_{\mathrm{I}}=140 \mathrm{~nm}$, tilt angle $=0^{\circ}$. (b) Dose $=1.1 \times 10^{13} / \mathrm{cm}^{2}, w_{\mathrm{I}}=250 \mathrm{~nm}$, tilt angle $=0$. The effect mode index change $\Delta n_{\text {eff }}$ is calculated at a reverse bias of $10 \mathrm{~V}$.

Fig. 4 presents the performance of the modulator versus the energy and the tilt angle of the $\mathrm{P}+$ and $\mathrm{N}+$ implantations. In our simulation the implantation energy of $\mathrm{P}$ is determined by that of $\mathrm{B}$ to situate their concentration peaks at the same horizontal level, so only the B energy is specified in Fig. 4. A tilt angle means the wafer rotates $\theta$ degrees around the $z$ axis of Fig. 1 while the ions are incident vertically. The rotations are anti-clockwise and clockwise for the $\mathrm{P}+$ and $\mathrm{N}+$ implantations respectively. Enhancing the implantation energy increases the standard deviation of the projected range [18]. A broader ion distribution overlaps better with the optical mode, so the modulation efficiency in Fig. 4(a) rises at first. Energy beyond $30 \mathrm{keV}$ would overshot ions into the bottom oxide layer, thus reduces the doping concentration and weakens the modulation efficiency. It is intriguing to find in Fig. 4(b) that a small tilt angle $\left(<6^{\circ}\right)$ reduces the optical loss remarkably almost without affecting the modulation efficiency. The reason is that a tilt angle can reduce the effective irradiated area at the bottom of the implantation window due to a shade effect. The effective irradiated area for a tilt angle of $\theta$ is $w_{\text {eff }}=w_{\mathrm{I}}-H \tan \theta$, where $w_{\mathrm{I}}$ and $H$ denote the width of the implantation window and the thickness of the implantation mask respectively. As we have elaborated in section II, the desirable width of the $\mathrm{P}+$ and $\mathrm{N}+$

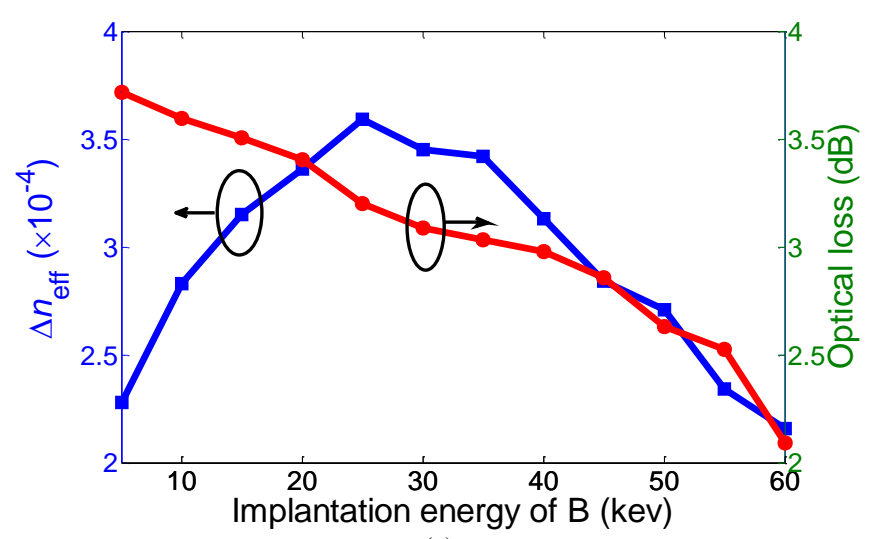

(a)

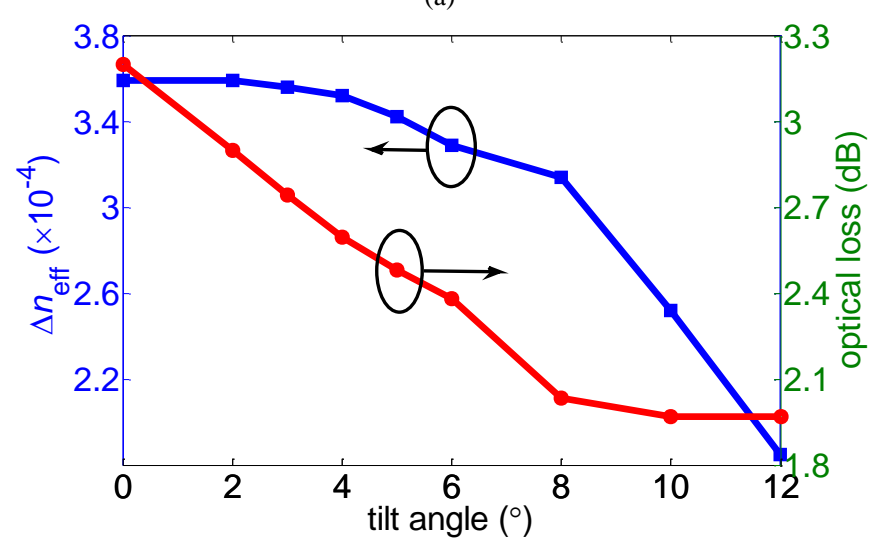

(b)

Fig. 4. Influences of (a) the implantation energy and (b) the tilt angle on the performance of the phase shifter. (a) tilt angle $=0^{\circ}$, (b) energy $=25 \mathrm{kev}$ (B). Other parameters in simulation are: $\mathrm{P}+(-0.12 \mathrm{~nm}, 0.02 \mathrm{~nm}), \mathrm{N}+(0.06 \mathrm{~nm}, 0.2$ $\mathrm{nm})$, Dose $=2.2 \times 10^{13} / \mathrm{cm}^{2}$

doped regions is $84.2 \mathrm{~nm}$. However, the ultimate width is $w_{\mathrm{I}}=140 \mathrm{~nm}$ due to the limitation of the practical lithograph. In our simulation the implantation mask thickness is $H=500 \mathrm{~nm}$, thus the final irradiated width is $w_{\text {eff }}=87.4 \mathrm{~nm}$ for a tilt angle of 6 . This value is quite close to the desired width of $84.2 \mathrm{~nm}$, so a simple calculation about $w_{\text {eff }}$ can well explain the curve in Fig. 4(b). According to the simulation result, we conclude that the optimal implantation energy and tilt angle for our structure are $25 \mathrm{kev}(\mathrm{B})$ and 4 respectively.

Up to now we have optimized the implantation condition for the active region. For the ion implantations in the $\mathrm{N}, \mathrm{P}, \mathrm{N}++$ and $\mathrm{P}++$ regions, we directly adopt the conditions listed in Table. I. The characteristic of the final phase shifter is presented in Fig. 2(a): its effective mode index shift at a reverse bias of $10 \mathrm{v}$ is $\Delta n_{\text {eff }}=0.0035$, which leads to a figure of merit of $2.2 \mathrm{~V} \cdot \mathrm{cm}$; the capacitance and the internal resistance per unit length at a reverse bias of $5 \mathrm{~V}$ are $0.205 \mathrm{fF} / \mu \mathrm{m}$ and $12 \mathrm{k} \Omega \cdot \mu \mathrm{m}$ respectively. The evolution of the extinction ratio with the phase shifter length is shown in Fig. 2(b), which indicates that the extinction ratio can be improved by $4.6 \mathrm{~dB}$ after the implantation condition optimization. Here we give an explanation about the implantation conditions of the $\mathrm{N}$ and $\mathrm{P}$ doped areas. The two areas are outside the active region, so most of their implantation parameters have little influence on the modulation except the dose. Enhancing the doping dose in 
the $\mathrm{N}$ and $\mathrm{P}$ regions can reduce the internal resistance and increase the optical loss, so a compromise should be made about this parameter. According to the simulation result listed above, the total internal resistance and capacitance of a $0.5 \mathrm{~mm}$ phase shifter are $24 \Omega$ and $0.102 \mathrm{pF}$ respectively. Since the output impedance of the RF power supply is $50 \Omega$, the 3 -dB bandwidth given by the formula $1 /(2 \pi R C)$ is $21.1 \mathrm{GHz}$ provided that a lumped electrode is applied. Compared with the reported modulators of the same phase shifter length [12], [19], the bandwidth of our structure is competitive. The internal resistance doesn't limit the modulation bandwidth, so there is no need to reduce it with the penalty of increasing the optical loss.

\section{Simplified DOPING PATTERN}

The doping pattern in Fig. 1(a) consists of 6 different doped regions, so 6 ion implantation steps are required. In order to save the fabrication cost, we hope to reduce the implantation steps under the premise of not deteriorating the preformance of the device. This can be achieved by the structure presented in Fig. 5(a). The two areas between the active and the metal contacting regions are alternately doped along the longitudinal direction with a period of $H$, so their equivalent implantation doses are the ratio of the practical doses to the filling factor of the doped channels. These doped channels are implanted simultaneously with the active region to save 2 implantation steps. We can tune the internal resistance by changing the filling factor. According to the simulation result in section III, a proper value of the filling factor can be 0.14. Another geometric parameter need to be defined is the period $H$, which can affect the intrinsic response time of the modulator. The intrinsic response time is the time required for carriers to be swept out and to come back into the active region. It can be characterized by the current response of the device to a voltage pulse. Extending the period $H$ leads to a longer route for the movement of free carriers, so it would increase the intrinsic response time. This point is evident in Fig. 5(b), which depicts transient current evolution with the time. The driven signal is a $10 \mathrm{~V}$ voltage pulse with a ramp time of $2 \mathrm{ps}$. Since a complete three-dimensional calculation requires too much simulation resources, the transient simulation here only calculates the two-dimensional planar structure in Fig. 5(a). The transient current response in Fig. 5(b) is defined as the current density per unit length along the direction perpendicular to the substrate for a $2.2 \mathrm{~mm}$ phase shifter, thus the final driving current is the product of the current density and the height of the rib waveguide, which is $220 \mathrm{~nm}$. Then the peak value of the driving current in Fig. 5(b) is several hundred milliamps, which coincides with previous reports [8]. The current response in Fig. 5(b) is faster at the rising edge of the voltage pulse than that at the trailing edge, so the cutoff frequency limited by the intrinsic response time is $f=0.35 / T_{\mathrm{t}}[8]$, where $T_{\mathrm{t}}$ is the response time of the current at the trailing edge of the voltage pulse. The response time is defined as the duration during which the current decreases from $90 \%$ to $10 \%$ of its peak value. In Fig. 5(b), $T_{\mathrm{t}}$ increases from $12.6 \mathrm{ps}$ to $24.4 \mathrm{ps}$ as the period $H$ grows

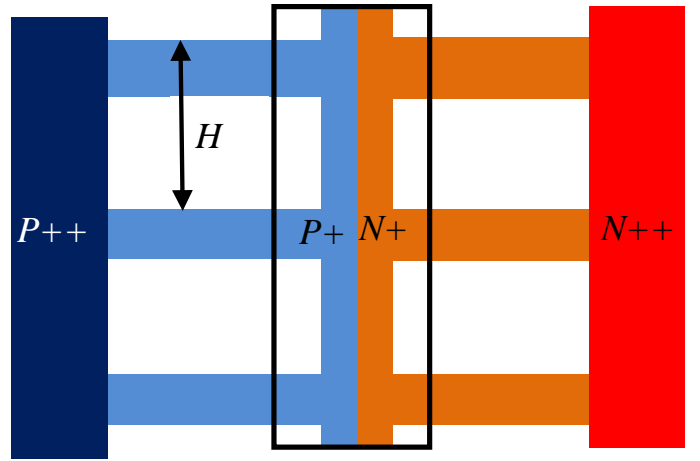

(a)

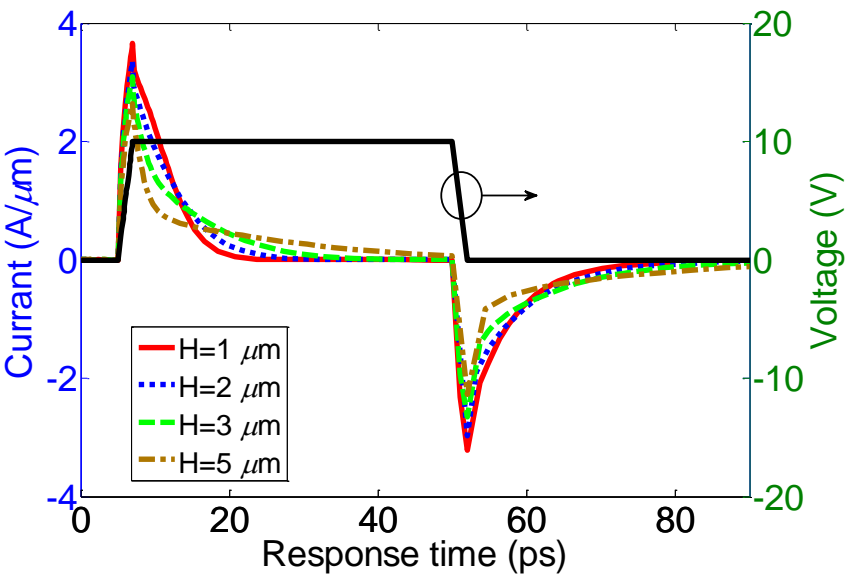

(b)

Fig. 5. Top view of the simplified doping pattern (a) and its current response to a voltage pulse (b). The black outline in (a) delineates the position of the rib waveguide.

from $1 \mu \mathrm{m}$ to $5 \mu \mathrm{m}$, thus the cutoff frequency falls from 27.8 $\mathrm{GHz}$ to $14.34 \mathrm{GHz}$. Since there are other limits for the modulation speed such as the RC constant, we suggest the period $H$ should not exceed $5 \mu \mathrm{m}$ for a high speed operation.

\section{CONCLUSION}

In this paper, we study the influence of the ion implantation condition on the performance of depletion-type silicon optical modulators. Through a comprehensive simulation about implantation parameters, such as the position, the energy and the tilt angle, the extinction ratio of final Mach-Zehnder modulator is enhanced by $4.6 \mathrm{~dB}$, and its insertion loss is reduced from $3 \mathrm{~dB}$ to $2.47 \mathrm{~dB}$. The improvement is achieved mainly by two approaches: at first, a gap between two specific implantation windows alleviates the detriment to the modulation efficiency caused by the lateral straggling of implanted ions. Secondly a tilt angle can relax the line width limit of the practical lithography on implantation windows, the final active region width can be slimmer than that defined in the implantation mask. Any extra optical loss at high doping concentrations can be minimized by using a tilt angle to equalize the active and the carrier depletion regions. Although the discussion in this paper is based on an ordinary lateral p-n junction based modulator, we believe that the technique in this paper can also be applied to other doping patterns as long as the optical modulation relies on carrier depletion. For example, if a 
vertical junction is applied instead of the lateral junction [19], then due to the ion straggling in the direction perpendicular to the wafer surface, it is also necessary to separate the two distribution peaks of $p$ and $n$ type dopants by a gap in this direction. Of course, the gap now is determined by the implantation energy rather than the implantation position. A specific simulation analogical to that presented in this paper can give the optimal implantation energy. In addition the doping pattern in Fig. 5(a) can also be used to reduce the unnecessary optical loss for the vertical junction based modulator.

\section{REFERENCES}

[1] R. S. Jacobsen, K. N. Andersen, P. I. Borel, J. F. Pedersen, L. H. Frandsen, O. Hansen and et al, "strained silicon as a new electro-optic material," Nature., vol. 441, no. 5, pp. 199-202, May. 2006.

[2] R. A. Soref and B. R. Bennett, "Electrooptical effects in silicon," IEEE J. Quantum Electron., vol. 23, no. 1, pp. 123-129, Jan. 1987.

[3] H. F. Zhou, Y. Zhao, W. J. Wang, J. Y. Yang, M. H. Wang, and X. Q. Jiang, "Performance influence of carrier absorption to the Mach-Zehnder-interference based silicon optical switches," Opt. Express., vol. 17, no. 9, pp. 7043-7051, Apr. 2009.

[4] J. V. Campenhout, W. M. J. Green, S. Assefa, and Y. A. Vlasov, "Low-power, $2 \times 2$ silicon electro-optic switch with 110 -nm bandwidth for broadband reconfigurable optical networks," Opt. Express., vol. 17, no. 26, pp. 24020-24029, Dec. 2009.

[5] K. Preston, S. Manipatruni, A. Gondarenko, C. B. Poitras, and M. Lipson, "Deposited silicon high-speed integrated electro-optic modulator," Opt. Express., vol. 17, no. 7, pp. 5118-5124, Mar. 2009.

[6] L. Liao, D. S. Rubio, M. Morse, A. S. Liu, and D. Hodge, "High speed silicon Mach-Zehnder modulator," Opt. Express., vol. 13, no. 8, pp. 3129-3135, Apr. 2005.

[7] X. Chen, Y. S. Chen, Y. Zhao, W. Jiang, and R. Chen, "Capacitor-embedded $0.54 \mathrm{pJ} / \mathrm{bit}$ silicon-slot photonic crystal waveguide modulator," Opt. Lett., vol. 34, no. 5, pp. 602-604, Mar. 2009.

[8] Z. Y. Li, D. X. Xu, W. R. McKinnon, S. Janz, J. H. Schmid, and et al, "silicon waveguide modulator based on carrier depletion in periodically interleaved PN junations," Opt. Express., vol. 17, no. 18, pp. 15947-15958, Aug. 2009.

[9] J. W. Park, J. B. You, I. G. Kim and G. Kim, "High-modulation efficiency silicon Mach-Zehnder optical modulator based on carrier depletion in a PN diode," Opt. Express., vol. 17, no. 18, pp. 15520-15524, Aug. 2009.

[10] P. Dong, S. Liao, D. Feng, H. Liang, D. Zheng, and et al, "Low Vpp, ultralow-energy, compact, high-speed silicon electro-optic modulator," Opt. Express., vol. 17, no. 25, pp. 22484-22490, Dec. 2009.

[11] F. Y. Gardes, A. Brimont, P. Sanchis, G. Rasigade, D. Marris-Morini, and et al, "High-speed modulation of a compact silicon ring resonator based on a reverse-biased pn diode," Opt. Express., vol. 17, no. 24, pp. 21986-21991, Nov. 2009.

[12] S. J. Spector, M. W. Geis, G.-R.Zhou, M. E. Grein, F. Gan, and et al, "CMOS-compatible dual-output silicon modulator for analog signal processing," Opt. Express., vol. 16, no. 15, pp. 11027-11031, Jul. 2008.

[13] D. M. Morini, L. Vivien, J. M. Fédéli, E. Cassan, P. Lyan, and S. Laval, "Low loss and high speed silicon optical modulator based on a lateral carrier depletion structure," Opt. Express., vol. 16, no. 1, pp. 334-339, Jan. 2008.

[14] D. Taillaert, F. V. Laere, M. Ayre, W. Bogaerts, D. V. Thourhout, P. Bienstman and R. Baets, "Grating couplers for coupling between optical fibers and nanophotonic waveguides," Jpn. J. Appl. Phys., vol. 45, no. 8A, pp. 6071-6077, 2006

[15] S. M. Sze and K. K. Ng, Physics of semiconductor devices. New York: Wiley, 2007, pp. 79-90, 197-212.

[16] S. K. Selvaraja, P. Jaenen, W. Bogaerts, D. V. Thourhout, P. Dumon and R. Baets, "Fabrication of Photonic Wire and Crystal Circuits in Silicon-on-Insulator Using 193nm Optical Lithography," J. Lightw. Technol, vol. 27, no. 18, pp. 4076-4083, Sep. 2009.

[17] ISE Integrated Systems Engineering AG, Affolternstrasse 52, CH-8050 Zurich, Switzerland.

[18] K. Suzuki, R. Sudo, and M. Nagase, "Estimating lateral straggling of impurity profiles of ions implanted into crystalline silicon," IEEE Trans.
Electron Devices., vol. 48, no. 12, pp. 2803-2807, Dec. 2001.

[19] M. R. Watts, W. A. Zortman, D. C. Trotter, R. W. Young and A. L. Lentine, "Low-voltage, compact, depletion-mode, silicon Mach-Zehnder modulator," IEEE J. Select.Topics Quantum Electron., vol. 16, no. 1, pp. 159-164, Jan/Feb. 2010.

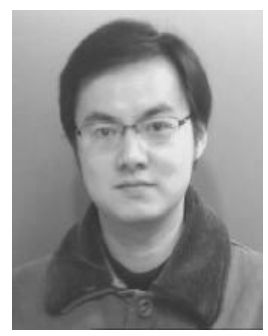

Hui Yu received the Bachelor's degree in electrical engineering from Zhejiang University, Hangzhou, China, in 2003, where he also obtained his $\mathrm{PhD}$ in electrical engineering in 2008.

$\mathrm{He}$ is currently a postdoc researcher with the Department of Information Technology, Ghent University, Gent, Belgium. His current interests include the modeling, design and fabrication of silicon active devices.

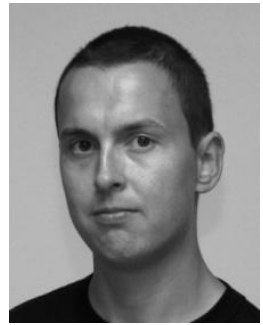

Wim Bogaerts (S'98 - M'05) completed his studies in engineering (applied physics) at Ghent University in 1998 and joined the department of information technology (INTEC) at both Ghent University and the Interuniversity Microelectronics Center (imec) where he received his $\mathrm{PhD}$ degree in 2004. In the photonics research group he specialized in the modeling, design and fabrication of nanophotonic components. Currently he coordinates the development of nanophotonic components in SOI in imec. He is a member of the IEEE Photonics Society (formerly Lasers and Electrooptics Society, LEOS) and the Optical Society of America (OSA).

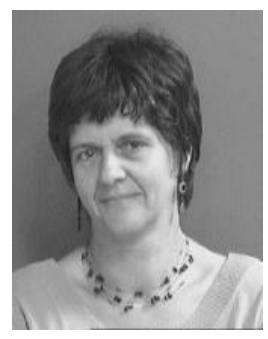

An De Keersgieter received the M.S. degree in physics from Ghent University, Belgium, in 1984. She joined imec in 1985, working on process modeling. She has been involved in modeling and simulation work for several European projects such as STORM, ADEQUAT, ACE, HUNT, ARTEMIS, NANOCMOS and PULLNANO. At present she is mainly focusing on process and device simulation and calibration of $65 \mathrm{~nm}$ and smaller CMOS technologies. Among fields of interest are strain-engineering and multi-gate devices. Next to this she is responsible for support of TCAD tools used at imec. She is a member of scientific committee (Modeling and Simulation) for the ESSDERC conference. 